PART 4:

\title{
THE NEXT DECADE
}




\title{
A roadmap towards habitable exoplanets by the Blue Dots Initiative
}

\author{
V. Coudé du Foresto ${ }^{1}$, for the Blue Dots participants \\ ${ }^{1}$ Observatoire de Paris - LESIA, Meudon, Paris, France \\ email: vincent.foresto@obspm.fr
}

\begin{abstract}
This paper is an abridged version of the report produced by the Blue Dots initiative, whose activities include the elaboration of a roadmap towards the spectroscopic characterization of habitable exoplanets. The full version of the Blue Dots report can be downloaded at http://www.blue-dots.net/spip.php?article105. While the roadmap will need to be updated regularly, it is expected that the methodology developed within Blue Dots will provide a durable framework for the elaboration of future revisions.
\end{abstract}

Keywords. planetary systems

\section{Introduction}

The Blue Dots initiative was created in 2008 to contribute towards building a community in Europe around the exoplanet theme, and to converge towards a strategy enabling a more coherent approach to Calls for Proposals in ground- and space-based projects. The scope of the initiative is science-oriented and not restricted to a particular detection technique.

The intiative gathers more than 180 scientists, mostly located in Europe, with additional participation from the US, Japan, and India. Participants are organized in different working groups covering the relevant science themes (Targets and their Environments, Formation and Evolution of Planetary Systems, Habitability Criteria, Observation of Planetary Atmospheres) and methods (Single Aperture Imaging, Multiple Aperture Imaging, Microlensing, Modelling Habitable Planets, Radial Velocities, Astrometry, Transits). The complete report and most of the material produced by the initiative can be found on its web site at http://www.blue-dots.net.

\section{Elements for a Roadmap}

The initial Blue Dots activity consisted of preparing a roadmap towards the detection and characterization of habitable exoplanets, recognizing that this ambitious goal will require several intermediate steps.

To achieve this a framework for discussion had to be created and several underlying principles were applied. First, science questions should drive the roadmap - techniques should be seen as tools to address these questions. Specific missions should be introduced as late as possible in the process if convergence is to be sought. We also felt it was important to clearly identify the points of consensus and the matters of debate. Points of consensus provide an opportunity for the community to send out a common message. Scientific debates are obviously healthy and useful when they can be organized in a way to help clarify the issues.

A roadmap should also find a way to get around the "pathfinder dilemma" which could be expressed this way: it is clear that the goal of spectroscopic characterization of the 
atmosphere of habitable planets, in search of biomarkers, will ultimately require one or more very ambitious and innovative missions. Those cannot meet the feasibility criteria as currently established by the space agencies, whose current trend is to select missions which are both low-risk and with an immediate science return. And the immediate science return of a more affordable demonstrator (one that would retire the risk on the bigger mission) does not necessarily meet the agency standards.

The frame of discussion required the creation of homogeneous grids that would cover the science that can can/should be achieved, and the methods that can be employed to achieve it. These are presented below.

\subsection{Science Potential Levels}

Following the lines of the step-by-step approach mentioned above, for a given class of objects the science potential level (SPL) of a technique is defined by its capacity:

* To carry out a statistical study of objects in a given class;

** To designate targets in the solar neighborhood for spectroscopic follow-up study;

*** To carry out a spectroscopic characterization of the object.

\subsection{Target Classes}

These classes are not meant to categorize objects according to their physical nature rather they group objects of similar detection difficulty. The five classes identified are (by order of increasing detection difficulty):

(a) Hot giant planets: these planets can be hot either because they are close to their host stars and highly irradiated, or because they are young;

(b) Other giant planets: these planets include the warm and cold gaseous giants, down to Neptune size;

(c) Hot telluric planets that might be hot, young or slightly more massive than the Earth (super-Earth), not necessarily located in the habitable zone neither around specific stars;

(d) Telluric planets in the habitable zone of M-type stars;

(e) Telluric planets in the habitable zone of solar-type stars.

\subsection{Observing Methods}

The methods employed for detecting and characterizing exoplanets can be classified in six large families, which are represented in the corresponding Blue Dots working groups:

- The microlensing method which consists in monitoring the photometry of distant stars in order to detect microlensing events.

- The transit photometry method which relies on measuring the relative change of the photometry of the star due to a primary eclipse (the planet transiting in front of the stellar photosphere) or to a secondary eclipse (the planet disappearing behind the star).

- The radial velocities method (RV) which relies on measuring the Doppler shift of star spectra with high precision in order to detect the reflex motion due to the presence of one or several planets. This method also includes timing techniques.

- The astrometry method whether in narrow-angle or globally consists in measuring the relative position of stars in order to detect the reflex motion due to the presence of orbiting planets.

- The single aperture imaging (SAI) technique includes all types of coronagraphic methods, including external occulters as well as imaging techniques using Fresnel lenses, in order to separate the direct light of the planets from the stellar light which is usually hidden. 
- The multiple aperture imaging (MAI) technique uses interferometric nulling, hypertelescopes, etc. to extract the direct light from the planet and to some extent the stellar light.

It is clearly understood that these methods are in many ways interrelated and complementary, inasmuch as observables obtained by one technique can often be interpreted only with the help of additional information from another technique. The reader is referred to the Blue Dots report for more details on each method, its current state of the art, the prospective performance, and the required R\&D efforts needed to achieve them.

\subsection{Project Scales}

Projects related to the different detection methods are listed and discussed in the Blue Dots report. It is recognized that those projects, which can be ground-based instruments or facilities, or spaceborne missions, can be of different scales. These are symbolized by different letter codes:

- E code: existing, or already programmed efforts on an existing facility;

- G code: projects which are not yet funded but whose effort is equivalent to that of a ground based instrument on an extremely large telescope ( $\simeq 30 \mathrm{M}$ euro, 5 -10 years);

- M code: projects whose effort is comparable to an ESA M-class mission ( $\simeq 450$ M euro);

- L code: flagship projects corresponding to an ESA L-class mission $(\simeq 650 \mathrm{M}$ euro, 10 15 years), or even larger projects (XL, $\geqslant 1$ G euro) carried out in worldwide collaboration where ESA's participation would be an L-size mission in itself. A typical time frame for such projects is $20+$ years.

\section{Producing Synthetic Grids}

With this approach, it is possible to produce a grid that provides a synthetic view of the potential of each family of detection methods for different classes of exoplanets (Figure 1). Another way to present the information is to produce a timeline grid (Figure 2) which presents the progression in observation capacity for each family of methods, as a function of the increasing size of the projects.

In the context of the goal of Blue Dots (spectroscopic characterization of habitable telluric exoplanets), a roadmap should be represented by the path of least effort from the current state of the art (the E-coded boxes, located mostly in the upper left of the grid of Figure 1) to where lies the goal of Blue Dots (SPL *** in the last two columns, or in the last column if one is only concerned with earth twins around solar twins).

\begin{tabular}{|c|c|c|c|c|c|}
\hline \multirow[b]{3}{*}{ Methods } & \multicolumn{5}{|c|}{ Planet classes } \\
\hline & \multirow[t]{2}{*}{$\begin{array}{l}\text { Hot Giant Planets } \\
\text { (young or hot) }\end{array}$} & \multirow{2}{*}{$\begin{array}{c}\text { Other Giant } \\
\text { Planets } \\
\text { (same as in Solar } \\
\text { System) }\end{array}$} & \multirow{2}{*}{$\begin{array}{l}\text { Hot Terrestrial } \\
\text { Planets } \\
\text { (hot, young or super- } \\
\text { Earth) }\end{array}$} & \multirow{2}{*}{$\begin{array}{l}\text { Telluric Planet in } \\
\text { habitable zone } \\
\text { around M-dwarfs }\end{array}$} & \multirow{2}{*}{$\begin{array}{c}\text { Telluric Planet in } \\
\text { habitable zone } \\
\text { around solar-type } \\
\text { stars }\end{array}$} \\
\hline & & & & & \\
\hline Microlensing & N/A & $\star E$ & $\star E$ & N/A & $\star M$ \\
\hline Transits & $\star \star \star E$ & 不完 $\mathbf{G}$ & $\star \star \star M$ & 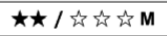 & $\star E$ \\
\hline Radial velocities & $\star \star E$ & $\star \star E$ & $\star \star E$ & $\star \star G$ & 额放 $\mathbf{G}$ \\
\hline Astrometry & $\star \star L$ & $\star \star E$ & $\star \star M$ & N/A & $\star \star L$ \\
\hline V imaging / coronagraphy (SAI) & $\star \star \star E$ & $\star \star \star G$ & $\star \star \star L$ & N/A & $\star \star \star L$ \\
\hline IR imaging / nulling (MAI) & $\star \star \star G$ & N/A & $\star \star \star L$ & $\star \star \star L$ & $\star \star \star L$ \\
\hline
\end{tabular}

Figure 1. Science potential levels ( $*$ : statistics, $* *$ : identification, $* * *$ : spectral characterization, $\mathrm{N} / \mathrm{A}$ when non applicable) of each family of detection methods for different classes of exoplanets. 


\begin{tabular}{|c|c|c|c|c|c|}
\hline \multirow[b]{2}{*}{ Methods } & \multirow[b]{2}{*}{$S P L$} & \multicolumn{4}{|c|}{ Project classes } \\
\hline & & Existing & Ground-based & M-class in space & L-XL class in space \\
\hline Microlensing & $\star$ & Giants & N/A & HZ telluric solar stars & N/A \\
\hline Transits & 太夫公放方 & Close giants & Other giants & All other terrestrials & N/A \\
\hline Radial velocities & $\star \star 太$ 公不 & Giant, close telluric & Habitable telluric & N/A & N/A \\
\hline Astrometry & $\star \star$ & Giants & N/A & Young telluric planets & Young + telluric in $\mathrm{HZ}$ \\
\hline V imaging / coronagraphy (SAI) & $\star \star \star$ & Young giants & Far giants & N/A & Telluric planets \\
\hline IR imaging / nulling (MAI) & $\star \star \star$ & N/A & Hot giants & N/A & All others \\
\hline
\end{tabular}

Figure 2. Timelines for various families of detection methods.

\section{Analysis}

One should first note the successes of the discipline and recognize that the E-coded boxes encompass some major achievements: spectroscopic characterization (SPL ***) has already been achieved on one class of exoplanets (hot giants), and a few telluric planets, albeit non habitable, have already been identified (SPL $* *$ ). If one considers the youth of our field (the first exoplanets around solar type stars were identified just 15 years ago), this is quite remarkable indeed, and if the trend continues it would give all reasons to be optimistic for the future.

One should also note that not every method is relevant for every class of object. This means that a roadmap necessarily has to rely on a portfolio of methods in order achieve the Blue Dots goal. Likewise, the timeline grid (Figure 2) shows that no single method has relevant projects at all scale levels. So, while some methods may be extremely productive now, other techniques need to be developed in order to take the relay when they are needed.

"Least effort" in the roadmap means that priority should be given, at each step towards the Blue Dots goal, to the method which enables to achieve it with the projects of the lowest scale.

Applying this principle leads to the conclusion that :

- SPL $*$ has been undertaken some years ago and results esentially from RV and microlensing surveys. The statistics of giants is quite known within a few AUs (typically $<4$ ) and the frequency of Super Earths is being investigated at short periods. Microlensing yields the statistics in the bulb down to a few Earth masses for separation of a few AU (0.5-5). There is clearly a need to extend this knowledge to longer periods (beyond the snow line) and to lower masses: this can be achieved by pushing radial velocity and direct imaging surveys.

- SPL $* *$ on habitable exoplanets should be carried out preferably from the ground by radial velocity. This is contingent to the acceptation that RV techniques are indeed capable to identify telluric habitable exoplanets. However, an exhaustive approach should involve at some stage an astrometric mission to be more resiliant with respect to the stellar parameters and to identify Earth masses around the nearby stars to be eventually characterized spectroscopically.

- Spectral characterization of telluric planets will certainly require space missions for two main reasons. First, the Earth atmosphere will make difficult (at low resolution) the analysis of other telluric planet atmospheres. Second, performance (stability, contrast) can be met more easily from space. In any case ground based telescopes and in particular ELTs will certainly contribute to this study but at a more modest level. That being said, SPL *** of close-in telluric planets around M stars should be attempted by transit photometry if it is indeed possible. Similarly, direct imaging at short wavelenghts could 
potentially achieve spectral characterization of telluric planets but for brighter/closer stars and larger separations (around 1-2 AU).

If one of these goals in the last item is achievable, this means that the goal of Blue Dots, at least in a selected sample of targets, can be achieved with a medium term project.

If not, and in any case for the solar type stars and Earth masses, spectroscopic characterization will require a flagship mission which will involve either an infrared interferometer, or an imager in the visible (and obviously preferentially both). It is not possible at this stage to prioritize these two options but we can work on collecting the elements that will help make the decision:

- Pursue the study of the exozodi issue to see how it impacts the detectability of habitable exoplanets in both cases ;

- Comparative system study for the two concepts in order to be able to compare performances/costs with an equivalent maturity level ;

- Pursue the identification of biomarkers and assess their detectability both in the infrared and the visible/near IR range.

Once these three steps are mastered, a flagship mission, possibly with global resources worldwide, in the decade 2020-2030 will follow naturally: the exoplanet community will be sufficiently knowledgeable to devise the best technological approach, to plan a sound observing program and to optimally exploit the data, especially spectroscopy.

\section{Conclusion}

No roadmap is carved in stone forever, and this is especially true with such a vibrant field as exoplanet science. It is likely to be revised every few years as the technology improves and new (possibly unexpected) results pour in. Blue Dots will continue to provide a forum and a framework to discuss these updates.

\section{Acknowledgements}

The material produced by Blue Dots comes from the contribution of all of the Blue Dots participants, and most notably its core team and working group coordinators which include Ignasi Ribas, Hans Zinnecker, Sebastian Wolf, Franck Selsis, Charles Cockell, Lisa Kaltenegger, Giovanna Tinetti, Anthony Boccaletti, Marc Ollivier, Jean-Philippe Beaulieu, Nuno Santos, Damien Segransan, Fabien Malbet, Alessandro Sozzeti, Ewa Szuszkiewicz, Helmut Lammer, Christoph Keller, Gerard van Belle, Szymon Gladysz, Chas Beichman, Hiroshi Shibai, Maxim Khodachenko, Gang Zhao, Abhijit Chakraborty, as well as $\simeq 180$ other scientists who participate at some level in the initiative. 